\title{
Position measuring interactions and the Heisenberg uncertainty principle
}

\author{
Masanao Ozawa \\ Graduate School of Information Sciences, Tôhoku University, Aoba-ku, Sendai 980-8579, Japan
}

Received 9 May 2002; received in revised form 15 May 2002; accepted 15 May 2002

Communicated by P.R. Holland

\begin{abstract}
An indirect measurement model is constructed for an approximately repeatable, precise position measuring apparatus that violates the assertion, sometimes called the Heisenberg uncertainty principle, that any position measuring apparatus with noise $\epsilon$ brings the momentum disturbance no less than $\hbar / 2 \epsilon$ in any input state of the apparatus. (C) 2002 Elsevier Science B.V. All rights reserved.
\end{abstract}

PACS: 03.65.Ta; 04.80.Nn; 03.67.-a

Keywords: Uncertainty principle; Quantum measurement; Noise; Disturbance; Quantum nondemolition measurement; Gravitational wave detection

\section{Introduction}

Measurements disturb microscopic objects inevitably. The problem still remains open as to how measurements disturb their objects. It is frequently claimed that if one measures position with noise $\epsilon$, the momentum is disturbed at least $\hbar / 2 \epsilon[1$, p. 230]. This claim is often called the Heisenberg uncertainty principle. The Heisenberg principle has been demonstrated typically by a thought experiment using the $\gamma$-ray microscope [2], and eventually accepted as a basic principle of quantum mechanics by many [3]. However, we have no known general proof for the Heisenberg principle.

On the other hand, we have another relation claiming that the product of the standard deviations of po-

E-mail address: ozawa@math.is.tohoku.ac.jp (M. Ozawa). sition and momentum in any quantum state is at least $\hbar / 2$. This relation, often called the Robertson uncertainty relation, has been generally proven from two basic principles of quantum mechanics, the Born statistical formula and the canonical commutation relation $[4,5]$, using the Schwarz inequality. However, this relation describes the limitation of preparing microscopic objects but has no direct relevance to the limitation of measurements on single systems [6-8].

In attempts of formulating the Heisenberg principle in a rigorous language, there have been serious conceptual confusions concerning the noise of measurement, as pointed out in Refs. [9,10] relative to a controversy as to whether the Heisenberg principle leads to a sensitivity limit of gravitational wave detection [11-14]. The purpose of this Letter is to reexamine the Heisenberg principle by giving rigorous definitions for noise and disturbance caused by general 
measuring interactions. Two models of position measuring interactions are examined in detail. The first one has been known for long [1] and used as a standard model of proposed quantum nondemolition measurements $[12,14]$. By this model we discuss the justification of our notions of noise and disturbance, and show how the Heisenberg principle dominates this model. Then, we modify the first model to obtain the second one which does not obey the Heisenberg principle. From this model, we conclude that we have a precise, approximately repeatable position measurement that violates the above formulation of the Heisenberg uncertainty principle.

\section{Indirect measurement models}

The disturbance on the object caused by a measurement can be attributed to an interaction, called the measuring interaction, between the object and the apparatus. In this letter, we consider indirect measurement models in which the measuring interactions are subject to the equations of motions in quantum mechanics $[15,16]$.

Let $\mathbf{A}(\mathbf{x})$ be a measuring apparatus with macroscopic output variable $\mathbf{x}$ to measure, possibly with some error, an observable $A$ of the object $\mathbf{S}$, a quantum system represented by a Hilbert space $\mathcal{H}$. The measuring interaction turns on at time $t$, the time of measure$m e n t$, and turns off at time $t+\Delta t$ between object $\mathbf{S}$ and apparatus $\mathbf{A}(\mathbf{x})$. We assume that the object and the apparatus do not interact each other before $t$ nor after $t+\Delta t$ and that the composite system $\mathbf{S}+\mathbf{A}(\mathbf{x})$ is isolated in the time interval $(t, t+\Delta t)$. The probe $\mathbf{P}$ is defined to be the minimal part of apparatus $\mathbf{A}(\mathbf{x})$ such that the composite system $\mathbf{S}+\mathbf{P}$ is isolated in the time interval $(t, t+\Delta t)$. By minimality, we naturally assume that probe $\mathbf{P}$ is a quantum system represented by a Hilbert space $\mathcal{K}$. Denote by $U$ the unitary operator on $\mathcal{H} \otimes \mathcal{K}$ representing the time evolution of $\mathbf{S}+\mathbf{P}$ for the time interval $(t, t+\Delta t)$.

At the time of measurement the object is supposed to be in an arbitrary (normalized vector) state $\psi$ and the probe is supposed to be prepared in a fixed (normalized vector) state $\xi$. Thus, the composite system $\mathbf{S}+\mathbf{P}$ is in the state $\psi \otimes \xi$ at time $t$. Just after the measuring interaction, the object is separated from the apparatus, and the probe is subjected to a local interaction with the subsequent stages of the apparatus. The last process is assumed to measure an observable $M$, called the probe observable, of the probe with arbitrary precision, and the output is represented by the value of the macroscopic output variable $\mathbf{x}$.

\section{Probability distribution of the output}

In the Heisenberg picture with the original state $\psi \otimes \xi$ at time $t$, we write $A(t)=A \otimes I, M(t)=$ $I \otimes M, A(t+\Delta t)=U^{\dagger}(A \otimes I) U$, and $M(t+\Delta t)=$ $U^{\dagger}(I \otimes M) U$. For any interval $\Delta$ in the real line, we denote by " $\mathbf{x} \in \Delta$ " the probabilistic event that the output of the measurement using apparatus $\mathbf{A}(\mathbf{x})$ is in $\Delta$. Since the output of this measurement is obtained by the measurement of the probe observable $M$ at time $t+\Delta t$, the probability distribution of the output variable $\mathbf{x}$ is given by

$\operatorname{Pr}\{\mathbf{x} \in \Delta\}=\left\langle E^{M(t+\Delta t)}(\Delta)\right\rangle$,

where $\langle\cdots\rangle$ stands for $\langle\psi \otimes \xi|\cdots| \psi \otimes \xi\rangle$ throughout this Letter, and where $E^{M(t+\Delta t)}(\Delta)$ stands for the spectral projection of the operator $M(t+\Delta t)$ corresponding to the interval $\Delta$.

\section{Noise in measurement}

We say that apparatus $\mathbf{A}(\mathbf{x})$ satisfies the Born statistical formula (BSF) for observable $A$ on input state $\psi$, if we have

$\operatorname{Pr}\{\mathbf{x} \in \Delta\}=\left\langle E^{A(t)}(\Delta)\right\rangle$.

We say that apparatus $\mathbf{A}(\mathbf{x})$ measures observable $A$ precisely, if $\mathbf{A}(\mathbf{x})$ satisfies the $\mathrm{BSF}$ for observable $A$ on every input state [17]. Otherwise, we consider apparatus $\mathbf{A}(\mathbf{x})$ to measure observable $A$ with some noise.

In order to quantify the noise, we introduce the noise operator $N(A)$ of apparatus $\mathbf{A}(\mathbf{x})$ for measuring $A$ defined by

$N(A)=M(t+\Delta t)-A(t)$.

The noise $\epsilon(A)$ of apparatus $\mathbf{A}(\mathbf{x})$ for measuring $A$ on input state $\psi$ is, then, defined by

$\epsilon(A)=\left\langle N(A)^{2}\right\rangle^{1 / 2}$. 
The noise $\epsilon(A)$ represents the root-mean-square error in the output of the measurement.

In order to clarify the meaning of the above definition, let us consider the case where the measured observable has a definite value just before the measurement, so that we assume $\psi=|A=a\rangle$. Then, we have

$N(A)|\psi \otimes \xi\rangle=[M(t+\Delta t)-a]|\psi \otimes \xi\rangle$

and

$\epsilon(A)=\left\langle[M(t+\Delta t)-a]^{2}\right\rangle^{1 / 2}$.

Thus, $\epsilon(A)$ stands for the root-mean-square deviation in the experimental output $M(t+\Delta t)$ from the value $a$ of observable $A$ taken at the time of measurement.

One of the fundamental properties of the noise is that precise apparatuses and noiseless apparatuses are equivalent notions, as ensured by the following theorem [18].

Theorem 1. Apparatus $\mathbf{A}(\mathbf{x})$ measures observable A precisely if and only if $\epsilon(A)=0$ on any input state $\psi$.

\section{Disturbance in measurement}

In this Letter, we assume that the apparatus carries out instantaneous measurements. In this case, we say that apparatus $\mathbf{A}(\mathbf{x})$ does not disturb the probability distribution of an observable $B$ of $\mathbf{S}$ on input state $\psi$, if

$\left\langle E^{B(t)}(\Delta)\right\rangle=\left\langle E^{B(t+\Delta t)}(\Delta)\right\rangle$,

for every interval $\Delta$, where we write $B(t)=B \otimes I$ and $B(t+\Delta t)=U^{\dagger}(B \otimes I) U$. We say that apparatus $\mathbf{A}(\mathbf{x})$ does not disturb observable $B$, if apparatus $\mathbf{A}(\mathbf{x})$ does not disturb the probability distribution of observable $B$ on any input state $\psi$ [16]. It was proven that apparatus $\mathbf{A}(\mathbf{x})$ does not disturb observable $B$ if and only if successive measurements of observables $A$ and $B$, using $\mathbf{A}(\mathbf{x})$ for $A$ measurement, satisfy the joint probability formula for simultaneous measurements [16].

In order to quantify the disturbance, we introduce the disturbance operator $D(B)$ of apparatus $\mathbf{A}(\mathbf{x})$ for observable $B$ defined by

$$
D(B)=B(t+\Delta t)-B(t) .
$$

The disturbance $\eta(B)$ of apparatus $\mathbf{A}(\mathbf{x})$ for observable $B$ on input state $\psi$ is, then, defined by

$\eta(B)=\left\langle D(B)^{2}\right\rangle^{1 / 2}$.

The disturbance $\eta(B)$ represents the root-mean-square deviation of the observable $B$ before and after the measuring interaction.

One of the fundamental properties of the disturbance is that apparatuses that do not disturb (the probability distribution of) the given observable and apparatuses with zero disturbance for that observable are equivalent notions, as ensured by the following theorem [18].

Theorem 2. Apparatus $\mathbf{A}(\mathbf{x})$ does not disturb observable $B$ if and only if $\eta(B)=0$ on any input state $\psi$.

\section{Von Neumann's model}

From now on, we consider the case where the object $\mathbf{S}$ is a one-dimensional mass with position $\hat{x}$ and momentum $\hat{p}_{x}$. Under general definitions given in the previous sections, we can rigorously formulate the Heisenberg uncertainty principle that any position measurement with noise $\epsilon$ disturbs the momentum at least $\hbar / 2 \epsilon$ by the relation

$\epsilon(\hat{x}) \eta\left(\hat{p}_{x}\right) \geqslant \frac{\hbar}{2}$.

Von Neumann [1, p. 443] introduced the following indirect measurement model of an approximate position measurement (see also Refs. [12,14,19]). The probe $\mathbf{P}$ is another one-dimensional mass with position $\hat{y}$ and momentum $\hat{p}_{y}$. The probe observable is taken to be position $\hat{y}$. The measuring interaction is given by

$\hat{H}=K \hat{x} \hat{p}_{y}$.

The coupling constant $K$ is so large that the free Hamiltonians can be neglected. The time duration $\Delta t$ of the measuring interaction is chosen so that $K \Delta t=1$. Then, the unitary operator of the time evolution of $\mathbf{S}+\mathbf{P}$ from $t$ to $t+\Delta t$ is given by

$U=\exp \left(\frac{-i}{\hbar} \hat{x} \hat{p}_{y}\right)$ 
Solving the Heisenberg equations of motion for $t<t+\tau<t+\Delta t$, we obtain

$\hat{x}(t+\tau)=\hat{x}(t)$,

$\hat{y}(t+\tau)=K \tau \hat{x}(t)+\hat{y}(t)$,

$\hat{p}_{x}(t+\tau)=\hat{p}_{x}(t)-K \tau \hat{p}_{y}(t)$,

$\hat{p}_{y}(t+\tau)=\hat{p}_{y}(t)$.

For $\tau=\Delta t=1 / K$, we have

$\hat{x}(t+\Delta t)=\hat{x}(t)$,

$\hat{y}(t+\Delta t)=\hat{x}(t)+\hat{y}(t)$,

$\hat{p}_{x}(t+\Delta t)=\hat{p}_{x}(t)-\hat{p}_{y}(t)$,

$\hat{p}_{y}(t+\Delta t)=\hat{p}_{y}(t)$.

It follows that the noise operator and the disturbance operator are given by

$N(\hat{x})=\hat{y}(t+\Delta t)-\hat{x}(t)=\hat{y}(t)$,

$D\left(\hat{p}_{x}\right)=\hat{p}_{x}(t+\Delta t)-\hat{p}_{x}(t)=-\hat{p}_{y}(t)$.

Thus, the position-measurement noise and the momentum disturbance are given by

$\epsilon(\hat{x})^{2}=\left\langle\hat{y}(t)^{2}\right\rangle$,

$\eta\left(\hat{p}_{x}\right)^{2}=\left\langle\hat{p}_{y}(t)^{2}\right\rangle$.

We denote by $\sigma(\hat{y})$ and $\sigma\left(\hat{p}_{y}\right)$ the standard deviations of the probe position and momentum at the time of measurement, respectively. By definition, we have

$\sigma(\hat{x})^{2}=\left\langle\hat{y}(t)^{2}\right\rangle-\langle\hat{y}(t)\rangle^{2} \leqslant \epsilon(\hat{x})^{2}$,

$\sigma\left(\hat{p}_{x}\right)^{2}=\left\langle\hat{p}_{y}(t)^{2}\right\rangle-\left\langle\hat{p}_{y}(t)\right\rangle^{2} \leqslant \eta\left(\hat{p}_{x}\right)^{2}$.

Thus, by the Robertson relation, we have

$\epsilon(\hat{x}) \eta\left(\hat{p}_{x}\right) \geqslant \sigma(\hat{y}) \sigma\left(\hat{p}_{y}\right) \geqslant \frac{\hbar}{2}$.

Therefore, we conclude that the von Neumann model obeys Eq. (10) as a consequence of the Robertson relation applied to the probe state just before measurement. In particular, this model represents a basic feature of the $\gamma$ ray microscope on the point that the trade-off between the noise and the disturbance arises from the fundamental physical limitation on preparing the probe. It might be expected that such a basic feature is shared by every model in a reasonable class of position measurements. However, the next model suggests that it is not the case.

\section{The model violating the Heisenberg uncertainty principle}

In what follows, we modify the measuring interaction of the von Neumann model to construct a model that violates Eq. (10). In this new model, the object, the probe, and the probe observables are the same systems and the same observable as the von Neumann model. The measuring interaction is taken to be [9]

$\hat{H}=\frac{K \pi}{3 \sqrt{3}}\left(2 \hat{x} \hat{p}_{y}-2 \hat{p}_{x} \hat{y}+\hat{x} \hat{p}_{x}-\hat{y} \hat{p}_{y}\right)$.

The coupling constant $K$ and the time duration $\Delta t$ are chosen as before so that $K \gg 1$ and $K \Delta t=1$. Then, the unitary operator $U$ is given by

$U=\exp \left[\frac{-i \pi}{3 \sqrt{3} \hbar}\left(2 \hat{x} \hat{p}_{y}-2 \hat{p}_{x} \hat{y}+\hat{x} \hat{p}_{x}-\hat{y} \hat{p}_{y}\right)\right]$.

Solving the Heisenberg equations of motion for $t<t+\tau<t+\Delta t$, we obtain

$$
\begin{aligned}
\hat{x}(t+\tau)= & \frac{2}{\sqrt{3}} \hat{x}(t) \sin \frac{(1+K \tau) \pi}{3} \\
& +\frac{-2}{\sqrt{3}} \hat{y}(t) \sin \frac{K \tau \pi}{3}, \\
\hat{y}(t+\tau)= & \frac{2}{\sqrt{3}} \hat{x}(t) \sin \frac{K \tau \pi}{3} \\
& +\frac{-2}{\sqrt{3}} \hat{y}(t) \sin \frac{(1-K \tau) \pi}{3}, \\
\hat{p}_{x}(t+\tau)= & \frac{-2}{\sqrt{3}} \hat{p}_{x}(t) \sin \frac{(1-K \tau) \pi}{3} \\
& +\frac{-2}{\sqrt{3}} \hat{p}_{y}(t) \sin \frac{K \tau \pi}{3}, \\
\hat{p}_{y}(t+\tau)= & \frac{2}{\sqrt{3}} \hat{p}_{x}(t) \sin \frac{K \tau \pi}{3} \\
& +\frac{2}{\sqrt{3}} \hat{p}_{y}(t) \sin \frac{(1+K \tau) \pi}{3} .
\end{aligned}
$$

For $\tau=\Delta t=1 / K$, we have

$$
\begin{aligned}
& \hat{x}(t+\Delta t)=\hat{x}(t)-\hat{y}(t), \\
& \hat{y}(t+\Delta t)=\hat{x}(t), \\
& \hat{p}_{x}(t+\Delta t)=-\hat{p}_{y}(t), \\
& \hat{p}_{y}(t+\Delta t)=\hat{p}_{x}(t)+\hat{p}_{y}(t) .
\end{aligned}
$$


It follows that the noise operator and the disturbance operator are given by

$$
\begin{aligned}
& N(\hat{x})=\hat{y}(t+\Delta t)-\hat{x}(t)=0, \\
& D\left(\hat{p}_{x}\right)=\hat{p}_{x}(t+\Delta t)-\hat{p}_{x}(t)=-\hat{p}_{y}(t)-\hat{p}_{x}(t) .
\end{aligned}
$$

Thus, the position-measurement noise and the momentum disturbance are given by

$$
\begin{aligned}
& \epsilon(\hat{x})=0, \\
& \begin{aligned}
\eta\left(\hat{p}_{x}\right)^{2} & =\left\langle\left[\hat{p}_{x}(t)+\hat{p}_{y}(t)\right]^{2}\right\rangle \\
& =\sigma\left(\hat{p}_{x}\right)^{2}+\sigma\left(\hat{p}_{y}\right)^{2}+\left[\left\langle\hat{p}_{x}(t)\right\rangle+\left\langle\hat{p}_{y}(t)\right\rangle\right]^{2} .
\end{aligned}
\end{aligned}
$$

Consequently, we have [20]

$\epsilon(\hat{x}) \eta\left(\hat{p}_{x}\right)=0$.

Therefore, our model obviously violates Eq. (10).

Taking advantage of the above model, we can refute the argument that the uncertainty principle generally leads to a general sensitivity limit, called the standard quantum limit, for monitoring free-mass position $[9,13]$.

If $\left\langle\hat{p}_{x}(t)^{2}\right\rangle \rightarrow 0$ and $\left\langle\hat{p}_{y}(t)^{2}\right\rangle \rightarrow 0$ (i.e., $\psi$ and $\xi$ tend to the momentum eigenstate with zero momentum) then we have even $\eta\left(\hat{p}_{x}(t)\right) \rightarrow 0$ with $\epsilon(\hat{x})=0$. Thus, we can measure position precisely without effectively disturbing momentum in a near momentum eigenstate; see Ref. [21] for detailed discussion on the quantum state reduction caused by the above model.

\section{Repeatability and uncertainty principle}

In formulating the canonical description of state changes caused by measurements, von Neumann required not only the preciseness of measurement but also that the measurement of an observable satisfy the repeatability hypothesis [1, p. 335]: If an observable is measured twice in succession in a system, then we get the same value each time. On the other hand, the von Neumann model (12) does not satisfy the preciseness nor the repeatability. One of the characteristic features of our model (29) is that it measures position precisely, but our model does not satisfy the repeatability hypothesis either. Thus, it is tempting to understand that our model circumvents the Heisenberg inequality (10) by paying the price of failing the repeatability.
Nevertheless, the following argument will show that such a view cannot be supported.

In the first place, it has been proven that the repeatability hypothesis can be satisfied only by measurements of purely discrete observables. Davies and Lewis [22] gave a mathematical formulation of the repeatability hypothesis in a form that is meaningful even for measurements of continuous observables, and yet conjectured that it can be satisfied only by measurements of purely discrete observable. This conjecture was actually proven affirmatively [17,23]. Thus, no precise position measurements satisfy the repeatability hypothesis.

Secondly, if we consider the approximate repeatability, our model satisfies any stringent requirement on approximate repeatability. In order to show this, we need to introduce the measure of approximate repeatability. Suppose that we measure the position of mass $\hat{x}$ in succession using two apparatuses described by equivalent indirect measurement models. Suppose that the first apparatus with probe $\hat{y}$ interacts with $\hat{x}$ in $(t, t+\Delta t)$ and that the second apparatus with probe $\hat{z}$ interacts with $\hat{x}$ in $(t+\Delta t, t+2 \Delta t)$. Then, for any positive number $\alpha$, the position measurement is called an $\alpha$ approximately repeatable, if the root-mean-square deviation between the first output $\hat{y}(t+\Delta t)$ and the second output $\hat{z}(t+2 \Delta t)$ is no more than $\alpha$ [19], i.e.,

$$
\begin{aligned}
& \langle\psi \otimes \xi \otimes \xi|[\hat{z}(t+2 \Delta t)-\hat{y}(t+\Delta)]^{2} \\
& \quad \times|\psi \otimes \xi \otimes \xi\rangle \leqslant \alpha^{2} .
\end{aligned}
$$

If the apparatuses are equivalent to our model (29), we have

$\hat{z}(t+2 \Delta t)=\hat{x}(t+\Delta t)=\hat{x}(t)-\hat{y}(t)$,

and hence

$$
\begin{aligned}
& \langle\psi \otimes \xi \otimes \xi|[\hat{z}(t+2 \Delta t)-\hat{y}(t+\Delta t)]^{2} \\
& \quad \times|\psi \otimes \xi \otimes \xi\rangle=\left\langle\hat{y}(t)^{2}\right\rangle .
\end{aligned}
$$

Thus, assuming $\langle\hat{y}(t)\rangle=0$, we can conclude that our model is $\sigma(\hat{y})$ approximately repeatable.

Therefore, we conclude that although we have no repeatable position measurements in general, for any small $\alpha>0$ we have a precise, $\alpha$ repeatable position measurement that violates the Heisenberg inequality (10). This suggests that how stringent conditions might be posed for a class of position measurements, 
we could find at least one model that violates the Heisenberg inequality (10) in that class.

\section{Concluding remarks}

In their discussion on the Heisenberg principle, Braginsky and Khalili [24, p. 65] claimed that if the object input state is near a momentum eigenstate, then the post-measurement position uncertainty, $\sigma(\hat{x})(t+\Delta t)$, will be equal to the noise, $\epsilon(\hat{x})$. This claim and the subsequent derivation of the Heisenberg inequality is incorrect, since our model shows that $\sigma(\hat{x})(t+\Delta t) \rightarrow \infty$ and $\epsilon(\hat{x})=0$ when $\psi$ goes to the momentum eigenstate $\left|\hat{p}_{x}=0\right\rangle$, so that they can never be close.

Braginsky and Khalili [24, p. 66] claimed also that all linear measurements, measurements closely connected to linear systems, obey the Heisenberg inequality. However, our unitary operator (29) can be realized by linear systems as follows. The Hamiltonian (28) of our model comprises simple linear couplings $\hat{x} \hat{p}_{y}$ and $\hat{p}_{x} \hat{y}$ and an extra term $\hat{x} \hat{p}_{x}-\hat{y} \hat{p}_{y}$, which might resist a simple linear realization. However, the extra term can be eliminated by the following mathematical relation [21]

$$
\begin{aligned}
& \exp \left[\frac{-i \pi}{3 \sqrt{3} \hbar}\left(2 \hat{x} \hat{p}_{y}-2 \hat{p}_{x} \hat{y}+\hat{x} \hat{p}_{x}-\hat{y} \hat{p}_{y}\right)\right] \\
& \quad=\exp \left(-\frac{i}{\hbar} \hat{x} \hat{p}_{y}\right) \exp \left(\frac{i}{\hbar} \hat{p}_{x} \hat{y}\right) .
\end{aligned}
$$

Thus, our measuring interaction, (29), is equivalent to the consecutive linear couplings $\hat{p}_{x} \hat{y}$ and $\hat{x} \hat{p}_{y}$ [21]. The interactions corresponding to $\hat{p}_{x} \hat{y}$ and $\hat{x} \hat{p}_{y}$ have been known as the back-action evading (BAE) measurement [25] and its conjugate. They have been experimentally realized in linear optics [26,27] with equivalent optical setting of quadrature measurements. According to the above, our model can be experimentally realized at least in an equivalent optical setting using current linear optical devices, a combination of two mutually conjugate back-action evading amplifiers.

In Ref. [16], it was proven that any measuring apparatus disturbs every observable not commuting with the measured observable. Our model (29) suggests, however, that the trade-off between noise and disturbance should be quantitatively represented by a more complex formula than the Heisenberg inequality (10). Instead of Eq. (10), our model (29) actually satisfies the following trade-off between the initial position uncertainty $\sigma(\hat{x})$ and the momentum disturbance $\eta\left(\hat{p}_{x}\right)$ as

$\sigma(\hat{x}) \eta\left(\hat{p}_{x}\right) \geqslant \frac{\hbar}{2}$.

A proof of the above relation runs as follows. From Eq. (36), we have

$\left[\hat{x}(t), D\left(\hat{p}_{x}\right)\right]=\left[\hat{x}(t),-\hat{p}_{x}(t)\right]=-i \hbar$.

Thus, by the Robertson relation, we have

$\sigma(\hat{x}) \eta\left(\hat{p}_{x}\right) \geqslant \frac{1}{2}\left|\left\langle\left[\hat{x}(t), D\left(\hat{p}_{x}\right)\right]\right\rangle\right|=\frac{\hbar}{2}$.

A universally valid trade-off relation for the noise in position, the disturbance in momentum, and the initial uncertainties of position and momentum extending relations (10) and (46) to all generalized measurements will be shown in a forthcoming paper.

\section{Acknowledgements}

This work was supported by the programme "R\&D on Quantum Tech.” of the MPHPT of Japan, by the CREST project of the JST, and by the Grant-in-Aid for Scientific Research of the JSPS.

\section{References}

[1] J. von Neumann, Mathematical Foundations of Quantum Mechanics, Princeton University Press, Princeton, NJ, 1955.

[2] W. Heisenberg, Z. Phys. 43 (1927) 172.

[3] M. Jammer, The Philosophy of Quantum Mechanics: The Interpretation of Quantum Mechanics in Historical Perspective, Wiley, New York, 1974.

[4] E.H. Kennard, Z. Phys. 44 (1927) 326.

[5] H.P. Robertson, Phys. Rev. 34 (1929) 163.

[6] L.E. Ballentine, Rev. Mod. Phys. 42 (1970) 358.

[7] K. Kraus, Phys. Rev. D 35 (1987) 3070.

[8] H. Martens, W. de Muynck, Found. Phys. 20 (1990) 355.

[9] M. Ozawa, Phys. Rev. Lett. 60 (1988) 385.

[10] M. Ozawa, Realization of measurement and the standard quantum limit, in: P. Tombesi, E.R. Pike (Eds.), Squeezed and Nonclassical Light, Plenum, New York, 1989, pp. 263-286.

[11] V.B. Braginsky, Yu.I. Vorontsov, Usp. Fiz. Nauk 114 (1974) 41, Sov. Phys. Usp. 17 (1975) 644 (in English).

[12] C.M. Caves et al., Rev. Mod. Phys. 52 (1980) 341.

[13] H.P. Yuen, Phys. Rev. Lett. 51 (1983) 719. 
[14] C.M. Caves, Phys. Rev. Lett. 54 (1985) 2465.

[15] M. Ozawa, Phys. Rev. A 62 (2000) 062101.

[16] M. Ozawa, Phys. Rev. A 63 (2001) 032109.

[17] M. Ozawa, J. Math. Phys. 25 (1984) 79.

[18] A proof will be given in a forthcoming paper.

[19] M. Ozawa, J. Math. Phys. 34 (1993) 5596.

[20] Mathematically, we assume here that state $\psi$ is in a common domain of $\hat{x}$ and $\hat{p}_{x}$, while state $\xi$ is in a common domain of $\hat{y}$ and $\hat{p}_{y}$.
[21] M. Ozawa, Phys. Lett. A 282 (2001) 336.

[22] E.B. Davies, J.T. Lewis, Commun. Math. Phys. 17 (1970) 239.

[23] M. Ozawa, Publ. Res. Inst. Math. Sci., Kyoto Univ. 21 (1985) 279.

[24] V.B. Braginsky, F.Ya. Khalili, Quantum Measurement, Cambridge University Press, Cambridge, 1992.

[25] B. Yurke, J. Opt. Soc. Am. B 2 (1985) 732.

[26] S.F. Pereira et al., Phys. Rev. Lett. 72 (1994) 214.

[27] K. Bencheikh et al., Phys. Rev. Lett. 75 (1995) 3422. 